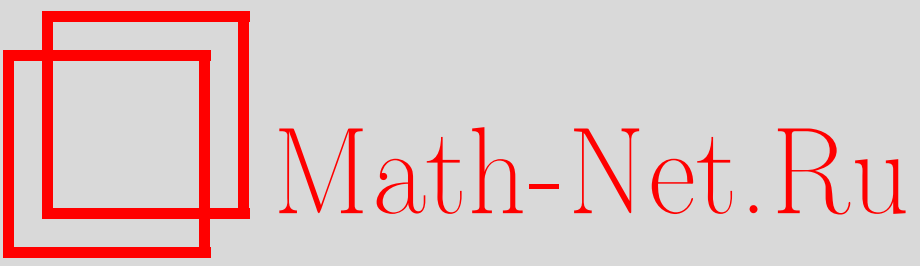

Д. В. Осипов, Отображение Кричевера и (полу)стабильные пучки на кривых, УМH, 1999, том 54, выпуск 3, 177-178

DOI: https://doi.org/10.4213/rm166

Использование Общероссийского математического портала Math-Net.Ru подразумевает, что вы прочитали и согласны с пользовательским соглашением

http: //www. mathnet.ru/rus/agreement

Параметры загрузки:

IP : 35.173 .219 .12

26 апреля 2023 г., 14:53:00 


\title{
ОТОБРАЖКЕНИЕ КРИЧЕВЕРА И (ПОЛУ)СТАБИЛЬНЫЕ ПУЧКИ НА КРИВЫХ
}

\author{
Д. В. Осипов
}

1. Пусть $k$ - произвольное поле, $V=k((z)) \oplus k((z)), V_{0}=k[[z]] \oplus k[[z]], \mu \in \mathbb{Z}$. Определим $\mathrm{Gr}^{\mu}(V)$ как множество $k$-векторных подпространств $W$ в $V$ таких, что $\operatorname{dim}_{k} V /\left(V_{0}+W\right)<\infty$, $\operatorname{dim}_{k} W \cap V_{0}<\infty$ и $\operatorname{dim}_{k} W \cap V_{0}-\operatorname{dim}_{k} V /\left(V_{0}+W\right)=\mu$. (См. подобные определения в [1]-[4].)

$\mathrm{Ha} \mathrm{Gr}^{\mu}(V)$ можно задать структуру бесконечномерного алгебраического многообразия, обобщающего конечномерное грассманово многообразие. (См. [1], где строится целая отделимая схема над $k, k$-точки которой совпадают с $\mathrm{Gr}^{\mu}(V)$.) На схеме $\mathrm{Gr}^{\mu}(V)$ можно определить линейное (детерминантное) расслоение Det такое, что для каждой $k$-точки $W \in \mathrm{Gr}^{\mu}(V)$ слой $\operatorname{Det}_{W}=\bigwedge^{\max }\left(W \cap V_{0}\right) \otimes \bigwedge^{\max }\left(V / W+V_{0}\right)^{*}($ см. [1], [3]).

Определим $S_{\mu}$ как множество последовательностей цельх чисел $\left\{s_{-\mu+1}, s_{-\mu+2}, \ldots\right\}$ таких, что: 1) эти последовательности строго убывающие; 2) $s_{k}=-k$ для $k \gg 0$.

Пусть Det* - двойственное линейное расслоение к расслоению Det. Отождествим $V$ и $k((z))$ при помощи следующего изоморфизма: $\left(\sum a_{i} z^{i}\right) \oplus\left(b_{i} z^{i}\right) \mapsto \sum\left(a_{i} z^{2 i}+b_{i} z^{2 i+1}\right)$. Тогда по любому $S \in S_{\mu}$ в [1], [3] строится сечение $\pi_{S}$ расслоения Det ${ }^{*}$ на $\mathrm{Gr}^{\mu}(V)$. При этом $W \in \mathrm{Gr}^{\mu}(V) \mapsto$ $\left\{\pi_{S}(W)\right\}$ определяет замкнутое вложение (плюккерово вложение) $\mathrm{Gr}^{\mu}(V) \hookrightarrow \mathbb{P}\left(\Pi\left(S_{\mu}\right)^{*}\right) \stackrel{\text { def }}{=}$ $\operatorname{Proj}\left(\operatorname{Sym}\left(\Pi\left(S_{\mu}\right)\right)\right)$, где $\Pi\left(S_{\mu}\right) \subset H^{0}\left(\mathrm{Gr}^{\mu}(V)\right.$, Det $\left.^{*}\right)$ - $k$-векторное пространство, порожденное глобальными сечениями $\left\{\pi_{S}: S \in S_{\mu}\right\}$. Последние, при этом, называются плюккеровыми координатами.

Любое $S=\left\{s_{-\mu+1}, s_{-\mu+2}, \ldots\right\} \in S_{\mu}$ однозначно определяет $S^{\prime}=\left\{s_{-\mu_{1}+1}^{\prime}, s_{-\mu_{1}+2}^{\prime}, \ldots\right\}$ $\in S_{\mu_{1}}$ и $S^{\prime \prime}=\left\{s_{-\mu_{2}+1}^{\prime \prime}, s_{-\mu_{2}+2}^{\prime \prime}, \ldots\right\} \in S_{\mu_{2}}$, так что множество $S=2 S^{\prime} \cup\left(2 S^{\prime \prime}+1\right)$, т.е. $\left\{s_{-\mu+1}, s_{-\mu+2}, \ldots\right\}=\left\{2 s_{-\mu_{1}+1}^{\prime}, 2 s_{-\mu_{1}+2}^{\prime}, \ldots\right\} \cup\left\{2 s_{-\mu_{2}+1}^{\prime \prime}+1,2 s_{-\mu_{2}+2}^{\prime \prime}+1, \ldots\right\}$. Положим $n(S) \stackrel{\text { def }}{=} \mu_{1}-\mu_{2}$.

Пусть $G=\mathrm{SL}(2, k[[z]])$. Естественное действие группы $G$ на $V$ индуцирует корректное действие $G$ на $\mathrm{Gr}^{\mu}(V)$.

2. Пусть 1) $C$ - неприводимая приведенная полная алгебраическая кривая над $k, 2) p \in C$ неособая $k$-рациональная точка, 3$) t_{p}$ - локальный параметр в точке $p$ (т.е. $\widehat{\mathscr{O}}_{p}=k\left[\left[t_{p}\right]\right]$, где $\widehat{\mathscr{O}}_{p}$ - пополнение локального кольца $\mathscr{O} p$ по максимальному идеалу), 4) $\mathscr{F}$ - когерентный пучок без кручения ранга 2 на $C, 5) e_{p}$ - базис свободного ранга 2 модуля $\widehat{\mathscr{F}}_{p}$ над $\widehat{\mathscr{O}}_{p}$, где $\widehat{\mathscr{F}}_{p} \stackrel{\text { def }}{=} \mathscr{F} \otimes_{\mathscr{O}_{C}}$ $\widehat{\mathscr{O}}_{p}$. Пусть эйлерова характеристика $\chi(\mathscr{F})=\mu$.

Можно определить отображение Кричевера $K$, которое каждому такому квинтету $\left(C, p, t_{p}, \mathscr{F}, e_{p}\right)$ сопоставляет замкнутую точку $K\left(C, p, t_{p}, \mathscr{F}, e_{p}\right) \in \mathrm{Gr}^{\mu}(V)$ (cм. [2], [4]): $\left(C, p, \mathscr{F}, t_{p}, e_{p}\right) \rightarrow H^{0}(C \backslash p, \mathscr{F}) \hookrightarrow H^{0}\left(\operatorname{Spec} \widehat{\mathscr{O}}_{p} \backslash p, \mathscr{F}\right)=\widehat{\mathscr{F}}_{p} \otimes_{\widehat{\mathscr{O}}_{p}} \widehat{K}_{p} \stackrel{e_{p}}{\rightarrow}\left(\widehat{\mathscr{O}}_{p} \oplus \widehat{\mathscr{O}}_{p}\right) \otimes_{\widehat{\mathscr{O}}_{p}}$ $\widehat{K}_{p} \stackrel{t_{p}}{\rightarrow} k((z)) \oplus k((z))$, где $\widehat{K}_{p^{-}}$поле частных кольца $\widehat{\mathscr{O}}_{p}$.

Напомним (см. [5]), что пучок $\mathscr{F}$ на $C$ называется (полу)стабильным, если для любого собственного подпучка $\mathscr{G} \subset \mathscr{F}: \chi(\mathscr{G})<\chi(\mathscr{F} / \mathscr{G})(\leqslant)$. Мы получаем следующую теорему.

Теорема 1. Пусть $W=K\left(C, p, t_{p}, \mathscr{F}, e_{p}\right)$. Пучок $\mathscr{F}$ не является (полу)стабильныц пучком тогда и только тогда, когда существует $g \in \mathrm{SL}(2, k[[z]])$, так что для любого $S \in S_{\mu}$ если $\pi_{S}(g W) \neq 0$, mo $n(S) \geqslant 0(>0)$.

ПримеР. На $\mathrm{Gr}^{\mu}(V)$ можно определить стратификацию - клеточное разбиение, индексированное множеством $S_{\mu}$ (см. [3]): $W \in \mathrm{Gr}^{\mu}(V)$ лежит в клетке $S=\left\{s_{-\mu+1}, s_{-\mu+2}, \ldots\right\}$, если $\pi_{S}(W) \neq 0$, и для любого $S^{\prime}=\left\{s_{-\mu+1}^{\prime}, s_{-\mu+2}^{\prime}, \ldots\right\} \pi_{S^{\prime}}(W) \neq 0$, если и только если

Работа выполнена при частичной финансовой поддержке Российского фонда фундаментальных исследований (гранты № № 96-15-96146, 96-15-96050). 
$s_{-\mu+1}^{\prime} \geqslant s_{-\mu+1}, s_{-\mu+2}^{\prime} \geqslant s_{-\mu+2}, \ldots$ Тогда если $W=K\left(C, p, t_{p}, \mathscr{F}, e_{p}\right)$ лежит в клетке $S \in S_{\mu}$ такой, что $S=2 S^{\prime} \cup\left(2 S^{\prime}+1\right)$, где $S^{\prime} \in S_{\mu / 2}$, то пучок $\mathscr{F}$ полустабилен $(\mu$ - четное).

3. Группа $G$, действуя на $W=K\left(C, p, t_{p}, \mathscr{F}, e_{p}\right) \in \mathrm{Gr}^{\mu}(V)$, меняет только базис $e_{p}$. И имеет место следуюшая лемма.

Лемма. Действие $G$ на $\mathrm{Gr}^{\mu}(V)$ поднимается до линейного действия $G$ на расслоение Det. При әтом, $k$-подпространство П $\left(S_{\mu}\right) \subset H^{0}\left(\mathrm{Gr}^{\mu}(V)\right.$, Det $\left.^{*}\right)-G$-инвариантно, $u\left(\begin{array}{cc}\lambda^{a} & 0 \\ 0 & \lambda^{-a}\end{array}\right)^{*}\left(\pi_{S}\right)=\lambda^{a \cdot n(S)} \pi_{S}$ для любъх $S \in S_{\mu}, \lambda \in k^{*}, a \in \mathbb{Z}$.

Поэтому в духе геометрической теории инвариантов (см. [5]) дадим следующие определения.

Для любого $W \in \mathrm{Gr}^{\mu}(V)$ пусть $\widehat{W}$ - некоторый не равный нулю элемент из $\Pi\left(S_{\mu}\right)^{*}$ такой, что образ $\widehat{W}$ в $\mathbb{P}\left(\Pi\left(S_{\mu}\right)^{*}\right)$ совпадает с образом $W$ при плюккеровом вложении. Для любых $\lambda \in k^{*}$, $g \in G$ пусть ${ }^{g} T(\lambda)=g\left(\begin{array}{cc}\lambda & 0 \\ 0 & \lambda^{-1}\end{array}\right) g^{-1}$. Пусть ${ }^{g} T=\left\{{ }^{g} T(\lambda), \lambda \in k^{*}\right\}$ - 1-параметрическая подгруппа: $k^{*} \rightarrow G$.

ОПредЕлЕниЕ. Замкнутая точка $W \in \mathrm{Gr}^{\mu}(V)$ - (полу)стабильная точка относительно 1-параметрической подгруппы ${ }^{g}$, если для $x=0$ и $x=\infty$ предел $\lim _{\lambda \rightarrow x} g_{T}(\lambda) \widehat{W}$ не сушествует (не существует или $\lim _{\lambda \rightarrow x} g_{T}(\lambda) \widehat{W} \neq 0$ ).

Теперь из леммы и теоремы 1 следует

TeOpema 2. Пусть $\left(C, p, t_{p}, \mathscr{F}, e_{p}\right)-$ квинтет, $\chi(\mathscr{F})=\mu$. Тогда пучок $\mathscr{F}$ (полу) стабилен на кривой $C$, если и только если $K\left(C, p, t_{p}, \mathscr{F}, e_{p}\right) \in \mathrm{Gr}^{\mu}(V)-($ полу $)$ стабильная точка относительно 1-параметрических подгрупп ${ }^{g} T$ для всех $g \in G$.

Автор выражает благодарность своему научному руководителю А. Н. Паршину за постоянное внимание к работе и ценные обсуждения.

\section{СПИСОК ЛИТЕРАТУРЫ}

[1] Álvarez Vázauez A., Muños Porras J. M., Plaza Martín F. J. The algebraic formalism of soliton equations over arbitrary base fields // Preprint alg-geom/9606009. [2] Mulase M. // Internat. J. Math. 1990. V. 1. № 3. P. 293-342. [3] Прессли Э., Сигал Г. Группы петель. М.: Мир, 1990. [4] Previato E., Wilson G. // Proc. Sympos. Pure Math. 1989. V. 49. P. 553-569. [5] Newstead P. E. Introduction to Moduli Problems and Orbit Spaces. Berlin: Springer-Verlag, 1978. (Tata Inst. of Fund. Research Lect. Notes on Math. and Phys. V. 51.) 